\title{
The influence of precursor gas pressure on structure and properties of fluoropolymer coatings by hot wire CVD
}

\author{
Alexey Safonov ${ }^{1, *}$, Veronica Sulyaeva ${ }^{2}$, Konstantin Kubrak $^{1}$, Sergey Starinsky ${ }^{1}$, and Nikolay \\ Timoshenko ${ }^{1}$ \\ ${ }^{1}$ Kutateladze Institute of Thermophysics SB RAS, 630090, Lavrentyev Str. 1, Novosibirsk, Russia \\ ${ }^{2}$ Nikolaev Institute of Inorganic Chemistry SB RAS, 630090, Lavrentyev Str. 3, Novosibirsk, Russia
}

\begin{abstract}
In this paper we investigated the deposition process of the fluoropolymer coatings by the method of Hot Wire Chemical Vapor Deposition. The dependence of precursor gas pressure on deposition rate, structure and properties of the formed film was investigated. The study has shown that the increasing of precursor gas pressure leads to change of the film structure from porous to continuous with globules and further to solid that have different wettability. Depending on the mode of deposition was obtained the fluoropolymer coating with different structure: the wetting angle changed from 60 to $170^{\circ}$. A mechanism of the formation of fluoropolymer coating was discussed.
\end{abstract}

\section{Introduction}

The surface of thin fluoropolymer films with high hydrophobic properties and chemical inertness can protect from corrosive environments, to intensify heat transfer at phase transitions, improve the surface antifrictional properties, etc. The structure of the film surface determines its properties. Thus, there is a need to develop methods to control of surface structure of the deposited coating.

The existing methods of deposition fluoropolymer films from the gas phase, such as plasma polymerization perfluoromonomer [1], magnetron sputtering [2], laser sputtering of the target, activation a gas precursor on the catalytic surface followed by deposition of a fluoropolymer on a substrate [3-5], require the improvement and the study of deposition processes.

The present work aims to study the possibilities of controlling the properties of fluoropolymer coatings deposited by Hot Wire Chemical Vapor Deposition (HWCVD) or CatCVD [4-6]. The method allows to control manages the structure of the resulting film by changing process parameters of deposition: the activating filament temperature [7], the substrate temperature, the precursor gas pressure, the mesh-substrate distance, etc.

\footnotetext{
* Corresponding author: safonov@itp.nsc.ru
} 


\section{Experimental setup and research techniques}

The scheme of experimental setup is shown in Fig. 1. The vacuum chamber for deposition of fluoropolymer coatings was pumped out by oil-free vacuum pump to the base pressure of $10^{-3}$ Torr. As the precursor hexafluoropropylene oxide $\mathrm{C}_{3} \mathrm{~F}_{6} \mathrm{O}$ was used. The precursor gas was fed through the flow controllers MKS 1179BX. The flow rate was constant and equal $20 \mathrm{sccm}$. In the center of the chamber the substrate was fixed. Above the substrate at a distance of $50 \mathrm{~mm}$ the catalytic activator from heated grid of nichrome wire was located. The temperature of the mesh $680{ }^{\circ} \mathrm{C}$ was monitored by electrical resistance and two contact chromel-alumel thermocouples. The substrate temperature was equal $30^{\circ} \mathrm{C}$ and was measured by the thermocouples. The pressure in the chamber was measured by a capacitive MKS Baratron sensor 623B and was regulated from 0.05 to 5 Torr with changing the pumping speed of the camera. As the substrates the plates of silicon (100) or copper with size $15 \times 15 \mathrm{~mm}^{2}$ were used.

The surface morphology and the thickness of the obtained coatings were determined by scanning electron microscopy (SEM) using the JEOL JSM-6700F microscope.

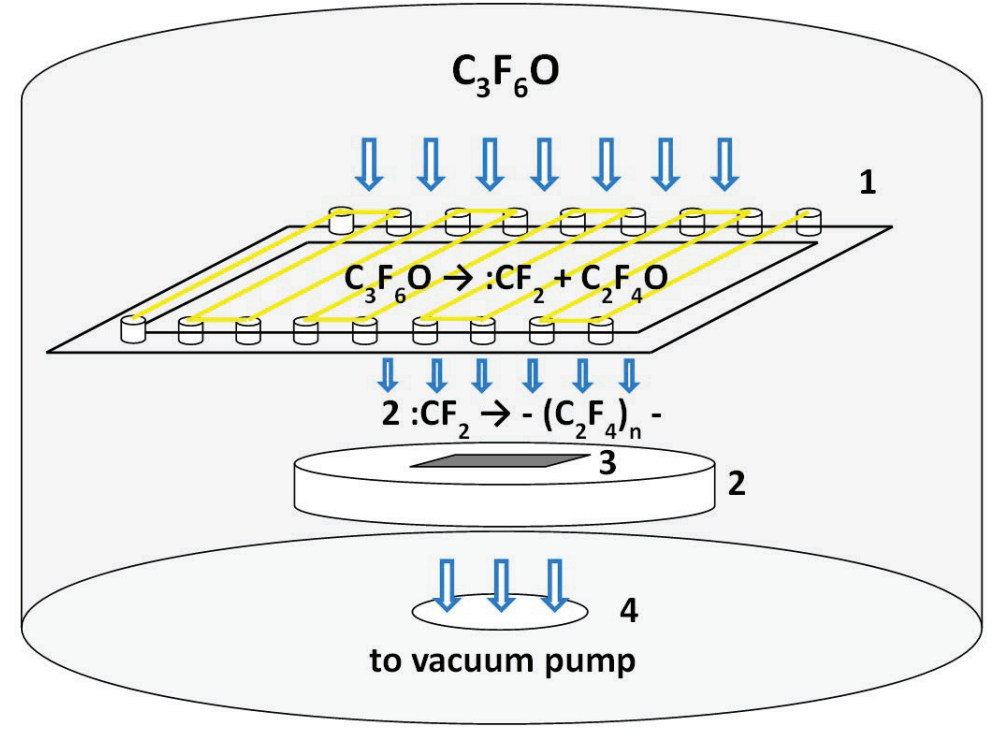

Fig. 1. The scheme of Hot Wire CVD method: 1 - wire activator of precursor gas; 2 - substrate holder with water cooling; 3 - silicon (100) and copper substrates; 4 - vacuum-pumping system.

\section{Results and discussion}

Fig. 2 shows the surface morphology of the obtained fluoropolymer coatings. In the SEM images are shown that at the precursor gas pressure of 0.1 Torr the films have a friable and porous structure. The cracks with length of several micrometers and a width of 5-10 nm were observed. With increasing pressure to 0.5 Torr the structure of the films varied, it become more dense and non-porous. The globule with size of $20-100 \mathrm{~nm}$ on the surface began to appear. A further increase of pressure led to an increasing of the number of globules on the surface and its agglomeration. At a pressure of about 5 Torr the globules finally coalesced into continuous film. 
The measurement of the thickness of the obtained coatings showed that at different precursor gas pressures during the coating deposition the formation rate varied substantially. The formation rate increased from 0.1 to $11.2 \mathrm{~nm} / \mathrm{s}$ at changing precursor gas pressure from 0.03 to 0.2 Torr, correspondingly. Further the formation rate reduced to 0.4 $\mathrm{nm} / \mathrm{s}$ at pressure of 1 Torr and stabilized at $0.1 \mathrm{~nm} / \mathrm{s}$ at 10 Torr.

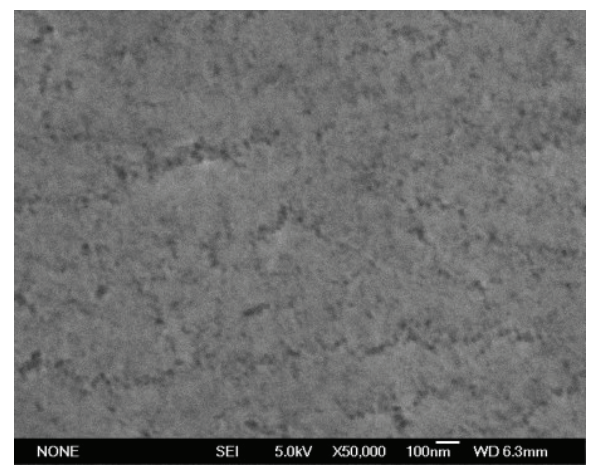

a

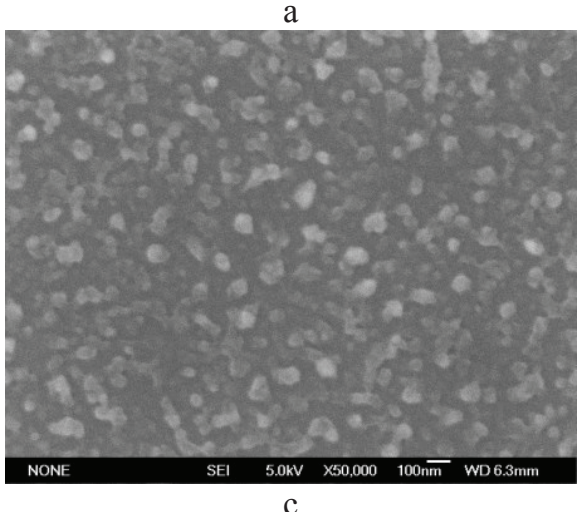

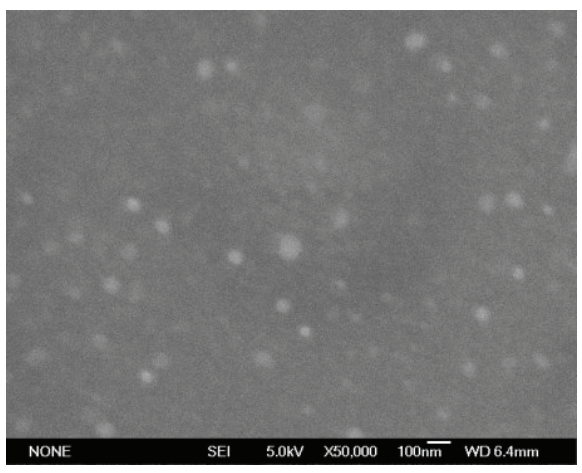

b

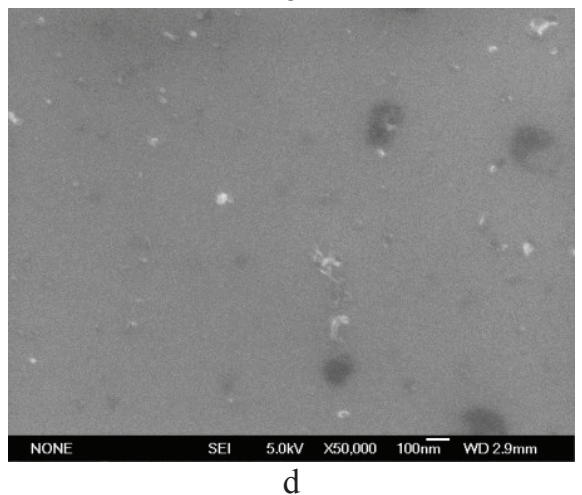

Fig. 2. SEM images of the fluoropolymer coatings surface obtained at different precursor gas pressure in the deposition process: $\mathrm{a}-0.1$ Torr; $\mathrm{b}-0.5$ Torr; $\mathrm{c}-1$ Torr; $\mathrm{d}-5$ Torr.

The model of formation process the fluoropolymer coating on the surface of the substrate is proposed to determine the influence of the main parameters on the deposition rate. The gas precursor molecules of the fluoropolymer passing through the mesh activated on its surface. The molecules $\mathrm{C}_{2} \mathrm{~F}_{4} \mathrm{O}$ and active radicals : $\mathrm{CF}_{2}$ [5] are formed. Further the radicals : $\mathrm{CF}_{2}$ are polymerized on the substrate surface. For the considered steady flow the number of activated particles is equal

$$
N_{a}=\frac{a P}{k T}
$$

where $a$ is the molecule activation coefficient. The formed active radicals can be deactivated by collisions. Let the coefficient of the deactivation on the free path $\lambda$ is equal $\Gamma \geq 1$. Then the number of active radicals at a distance $x$ from the surface activation will be equal

$$
N a x=\frac{a P / k T}{\Gamma^{\frac{\sqrt{2} \sigma P x}{k T}}}
$$


where $\sigma$ - section of collisions of molecules, $P$ and $T$ the pressure and temperature of the gas, correspondingly. The deposition rate obviously is proportional to the number of collisions of the active particles with the substrate surface. Thus, the deposition rate decreases exponentially with increasing distance $x$. The pressure dependence has a maximum at

$$
P=\frac{k T}{\sqrt{2} \sigma x \ln \Gamma}
$$

These simple estimates allow us to establish the qualitative dependence of the main parameters of the process on the deposition rate.

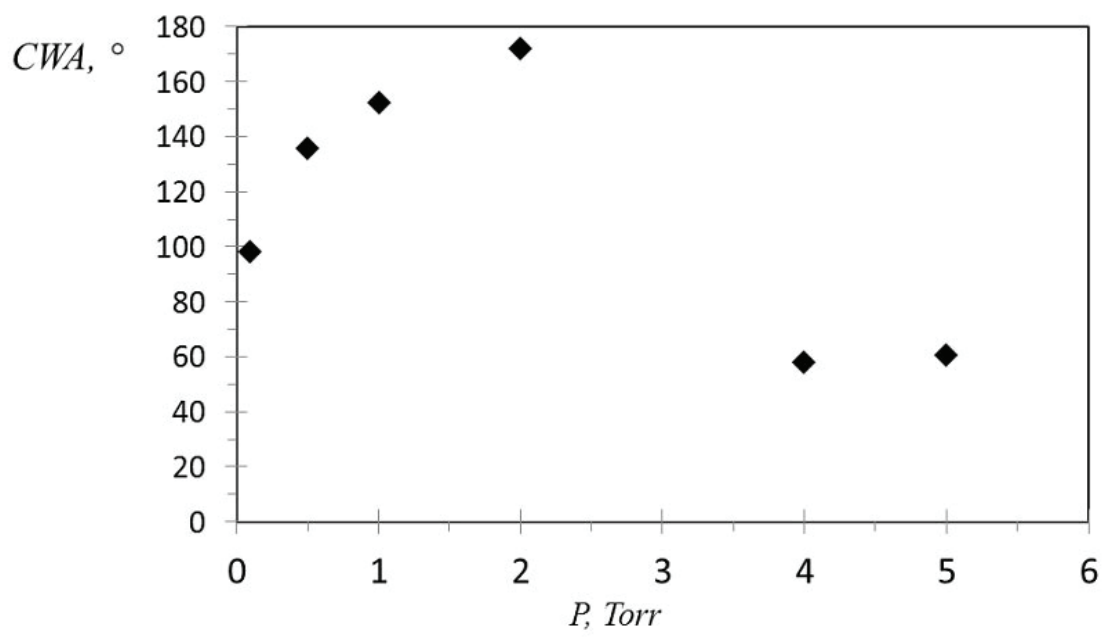

Fig. 3. The change of contact wetting angle depending on the precursor gas pressure in the deposition process.

The hydrophobic properties of the obtained fluoropolymer coatings was determined by measuring of the contact wetting angle (CWA) of its surface by a drop of water using a multifunctional modular device DSA 100 KRUSS. The results of the measurements are presented on the Fig. 3. It was found that the increasing of pressure in the range from 0.1 to 2 Torr lead to increasing the mean of contact wetting angle. The coatings had hydrophobic $\left(90^{\circ}<\right.$ CWA $\left.<150^{\circ}\right)$ and superhydrophobic $\left(150^{\circ}<\right.$ CWA $\left.<180^{\circ}\right)$ properties. Further increasing of precursor gas pressure leads to a significant decreasing in wetting angle of the obtained coatings. The coatings had hydrophilic $\left(30^{\circ}<\mathrm{CWA}<90^{\circ}\right)$ properties.

\section{Conclusions}

The change of deposition rate of fluoropolymer coatings depending on the precursor gas pressure has non-monotonic character with maximum at a defined mean of pressure. In the paper we propose a simple estimation of this relationship according to the number of deactivating the radical collisions.

The study has shown a significant effect of precursor gas pressure on the structure of formed fluoropolymer coating. The different structures of the obtained fluoropolymer coatings significantly affect its wetting properties. It leads to vary of the contact wetting angle of the obtained coatings from 60 to $170^{\circ}$.

This work was supported by the Grant of President of Russian Federation MK-5052.2016.8. 


\section{References}

1. H. Yasuda. Plasma Polymerization. (Academic Press, 1985)

2. W.-C. Wang. Plasma Process. Polym. 4, 88 (2007)

3. K. S. Lau, J. A. Caulfield, K. K. Gleason. Chem. Mater. 12, 3032 (2000)

4. K. S. Lau, H. G. Lewis, S. J. Limb, M. C. Kwan, K. K. Gleason. Thin Solid Films 395, 288 (2001)

5. A. K. Rebrov, A. I. Safonov, N. I. Timoshenko. Technical Physics Letters 35:5, 395 (2009)

6. K. Lau, Y. Mao, H. Lewis, S. Murthy, B. Olsen, L. Loo, K. Gleason. Thin Solid Films 501, 211 (2006)

7. A. Safonov, V. Sulyaeva, N. Timoshenko, E. Gatapova, O. Kabov, E. Kirichenko, A. Semenov. Matec 37, 01047 (2015) 